\title{
COMPARACIÓN DE DOS MÉTODOS ALTERNATIVOS AL POZO BARRENO PARA LA MEDICIÓN DE LA CONDUCTIVIDAD HIDRÁULICA SATURADA (Ks) EN UN ALFISOL $^{1}$
}

\author{
Marco Pfeiffer, Julio Haberland, Cristián Kremer, Oscar Seguel \\ Departamento de Ingeniería y Suelos, Facultad de Ciencias Agronómicas, Universidad de \\ Chile. Santa Rosa 11315, La Pintana, Santiago, Chile. Correspondencia: \\ marcopfeiffer@gmail.com
}

\section{Comparison of two methods to measure saturated hydraulic conductivity ( $\left.K_{s}\right)$ as alternative to auger hole method in an Alfisol}

Key words: Auger hole method, cilinder infiltrometer, constant head permeameter

\begin{abstract}
The following study compared two field methods and one laboratory method to measure saturated hydraulic conductivity (Ks) in the saturated phase of soil, all evaluated in a clayey loam soil with three replicates. The two field methods under study were the auger hole method (PB) and the cylinder infiltrometer (CI), the laboratory method was the constant head permeameter (PCC). Ks values delivered by the PCC method showed differences in magnitude $\left(1,03 \mathrm{~m} \mathrm{day}^{-1}\right)$ and a high variability $(\mathrm{CV}=249 \%)$, thus using these method is not recommended for soil with similar characteristics to the studied one. The PB and the CI methods showed a low variability $(\mathrm{CV}=39$ and $13 \%)$ and similar Ks values $\left(10,8\right.$ and $\left.7,1 \mathrm{~m} \mathrm{day}^{-1}\right)$, being recommended the use of both methods in soils with similar characteristics to the studied one. These methods are complementary, because of the $\mathrm{PB}$ requires the presence of a water level, meanwhile the CI requires the absence of these one.
\end{abstract}

Palabras clave: Pozo Barreno, Cilindro Infiltrómetro, Permeámetro de Carga constante

\section{RESUMEN}

El presente estudio evaluó el uso de alternativas al método del pozo barreno (PB) para la medición de conductividad hidráulica saturada $\left(\mathrm{K}_{\mathrm{s}}\right)$ en ausencia de un nivel freático. Se realizaron mediciones de $\mathrm{K}_{\mathrm{s}}$ en un suelo estratificado de textura superficial franco arcillo limosa fina sobre un sustrato de gravas y piedras mediante los métodos del pozo barreno (PB), el método del cilindro infiltrómetro (CI) y el método del permeámetro de carga constante (PCC). Los valores obtenidos con el método del PCC reflejaron una alta variabilidad $(\mathrm{CV}=249 \%)$ y diferencias de hasta un orden de magnitud en el valor de $\mathrm{K}_{\mathrm{s}}$ obtenido $\left(\mathrm{K}_{\mathrm{sx}}=1,03 \mathrm{~m} \mathrm{dia}^{-1}\right)$. Los métodos del PB y el CI presentaron coeficientes de

\footnotetext{
${ }^{1}$ Trabajo realizado como Memoria de Título para optar al Título Profesional de Ingeniero Agrónomo.
} 
variación bajos ( $\mathrm{CV}=39 \%$ y $\mathrm{CV}=13 \%$, respectivamente) para la medición de esta propiedad en terreno, lo que junto con la similitud en los valores obtenidos (10,8 y 7,1 m día $\left.\mathrm{a}^{-1}\right)$, permiten recomendar la utilización del CI como alternativa al PB en ausencia de nivel freático. Como contraparte el PCC, dada su variabilidad, no es recomendable como método alternativo en suelos con características similares al estudiado.

\section{INTRODUCCIÓN}

Se estima que en Chile, aproximadamente un $40 \%$ de los suelos potencialmente arables presentan problemas de drenaje (Alcayaga, 1989). La mayoría de estos suelos pueden ser habilitados para un uso intensivo mediante la realización de drenes de alivio, los cuales para su diseño requieren de la estimación de la conductividad hidráulica saturada del suelo $\left(\mathrm{K}_{\mathrm{s}}\right)$.

En su mayoría los métodos hidráulicos usados para la obtención de $\mathrm{K}_{\mathrm{s}}$ se basan en la ley de Darcy (1856):

$$
\mathrm{J}=\frac{Q}{A}=\frac{-\mathrm{Ks} \Delta \mathrm{H}}{\mathrm{L}}[1]
$$

Donde $\mathrm{J}$ ( $\mathrm{m} \mathrm{día}^{-1}$ ) es el flujo del fluido, Q $\left(\mathrm{m}^{3} \mathrm{dia}^{-1}\right)$ corresponde al flujo volumétrico de una sección de área $\mathrm{A}\left(\mathrm{m}^{2}\right)$ del material poroso, el coeficiente proporcional $\mathrm{K}_{\mathrm{S}}(\mathrm{m}$ $\mathrm{dia}^{-1}$ ) representa la descarga por unidad de área bajo un gradiente hidráulico unitario, y el gradiente hidráulico queda representado por $(\Delta \mathrm{H} / \mathrm{L})$, donde $\Delta \mathrm{H}(\mathrm{m})$ es la diferencia de carga hidráulica entre dos puntos y $\mathrm{L}(\mathrm{m})$ es el largo de la sección de suelo.

El método de medición de $\mathrm{K}_{\mathrm{s}}$ in situ más extendido es el del pozo barreno (PB), el cual requiere de la presencia de un nivel freático (NF) (Maasland, 1955; Bouwer and Jackson, 1974; Oosterban and Nijland, 1994).

En Chile, los suelos con problemas de drenaje están asociados a recargas estacionales, lo que genera fluctuaciones de los niveles freáticos a lo largo del año (Alcayaga, 1989; Salgado, 2000). Esto limita el tiempo disponible para las mediciones in situ mediante el PB, dejando por ende el desarrollo y término de proyectos de drenaje supeditados al éxito de esta medición.
Es por esto que se hace necesario evaluar un método que no requiera de la presencia de nivel freático para la medición de $\mathrm{K}_{\mathrm{S}}$, con el fin de dar una alternativa al método del pozo barreno (PB). Es así como, la presente investigación tuvo por objetivo evaluar los métodos del cilindro infiltrómetro(CI) y del permeámetro de carga constante (PCC) para ser utilizados como alternativa al método del PB en ausencia de nivel freático.

\section{MATERIALES Y MÉTODOS}

\section{Mediciones de $\mathbf{K}_{\mathrm{s}}$}

El estudio se realizó en el suelo La Obra, Aquultic Haploxeralf (CIREN, 1997), que corresponde a un suelo estratificado de origen aluvial, de textura superficial arcillo limosa, ubicado en la región del Maule. Se ubicó un sitio, el cual fue dividido en 3 parcelas (1,5 x 1,5 m cada una), con el fin de realizar las mediciones de $\mathrm{K}_{\text {s. }}$ Cada metodología analizada correspondió a un tratamiento, y cada tratamiento tuvo 3 repeticiones correspondientes a cada una de las parcelas.

En el mes de Agosto, cuando el nivel freático se encontraba a una profundidad promedio de $51 \mathrm{~cm}$, se realizaron mediciones con el método del pozo barreno. Para la realización de esta medición, un orificio es creado en el suelo mediante un barreno (6 $\mathrm{cm}$ de diámetro) hasta llegar a una determinada distancia bajo el nivel freático. Cuando el agua en el pozo entra en equilibrio con el nivel freático circundante, parte de esta es removida. El agua subterránea comienza entonces a filtrar hacia el orificio a una tasa determinada, la cual es medida. La conductividad hidráulica del suelo es 
obtenida mediante una fórmula que describe la relación existente entre la tasa de recuperación, las condiciones del agua subterránea y la geometría del orificio (para mayor detalle consultar Salgado, 2000).

Este método entrega un valor que corresponde a un promedio ponderado de la sección del perfil interceptada por el pozo que se encuentra bajo el nivel freático. Se realizaron 3 pozos mediante un barreno de $6 \mathrm{~cm}$ de diámetro, uno en cada parcela, y en cada pozo se realizaron 3 mediciones correspondientes a las submuestras de cada repetición. Los pozos se encontraban a $2 \mathrm{~m}$ de distancia entre sí. Entre cada repetición se esperó que el nivel freático se estabilizara a su nivel original.

La ecuación utilizada en este estudio para el método del pozo barreno corresponde a la ecuación de Hooghoudt (1936):

$$
\mathrm{K}=\frac{r S}{(2 H+r) \Delta t} \ln \frac{h_{0}}{h_{1}}[2]
$$

$\mathrm{y}:$

$$
\mathrm{S}=\frac{r H}{0,19}
$$

Cuadro 1. Propiedades Morfológicas del Perfil estudiado

\begin{tabular}{|c|c|}
\hline & Características Físicas y Morfológicas del Pedón: \\
\hline $\begin{array}{c}\text { 0 -20 } \\
\text { Ap }\end{array}$ & $\begin{array}{l}\text { Pardo amarillento ( } 10 \text { YR } 5 / 3) \text { en seco, pardo oscuro (10 YR } 3 / 3) \text { en } \\
\text { húmedo; franco arcillo limosa; plástico, ligeramente adhesivo; firme, } \\
\text { duro; bloques subangulares finos, medios y gruesos moderados; poros } \\
\text { finos comunes, gruesos escasos; raíces finas comunes; concreciones de } \\
\text { Fe y Mn escasos. Límite lineal claro }\end{array}$ \\
\hline $\begin{array}{l}20-60 \\
\text { Bw }\end{array}$ & $\begin{array}{l}\text { Pardo grisáceo ( } 10 \text { YR } 4 / 2) \text { en un } 60 \% \text { y pardo }(7.5 \text { YR } 4 / 6) \text { en un } 40 \% \text {, } \\
\text { ambos en húmedo, rasgos redox en líneas verticales; franco arcillo } \\
\text { limosa; plástico, ligeramente adhesivo; friable; bloques subangulares } \\
\text { finos, medios y gruesos moderados; poros muy finos, finos abundantes, } \\
\text { medios comunes, gruesos ocasionales; raíces finas escasas; concreciones } \\
\text { de Fe y Mn abundantes; abundante actividad biológica, presencia de } \\
\text { crotovinas, coprolitos y galerias de microfauna. Límite lineal gradual. }\end{array}$ \\
\hline $\begin{array}{l}60-80 \\
\text { BC }\end{array}$ & $\begin{array}{l}\text { Pardo ( } 7.5 \text { YR 4/6) en un } 80 \% \text {, pardo amarillento ( } 10 \text { YR } 4 / 3 \text { ) en un } \\
20 \% \text {; franco limosa; ligeramente plástico, ligeramente adhesivo; friable; } \\
\text { masivo; poros muy finos comunes, finos, medios y gruesos abundantes, } \\
\text { muy gruesos escasos; cutanes de Fe cubriendo los poros finos; Límite } \\
\text { lineal abrupto. }\end{array}$ \\
\hline $\begin{array}{l}80-110 \\
2 C_{1}\end{array}$ & $\begin{array}{l}\text { Estrata de guijarros y piedras redondeadas en mas de un } 90 \% \text {, en una } \\
\text { matriz areno francosa. Límite lineal claro. }\end{array}$ \\
\hline $\begin{array}{l}110-120 \\
2 C_{2}\end{array}$ & $\begin{array}{l}\text { Límite quebrado abrupto consistente en arena gruesa, la zona de } \\
\text { transición corresponde a la estrata de piedras. }\end{array}$ \\
\hline $\begin{array}{c}110-+ \\
2 C_{3}\end{array}$ & $\begin{array}{l}\text { Estrata de guijarros y piedras redondeadas en mas de un } 90 \% \text {, en una } \\
\text { matriz areno francosa }\end{array}$ \\
\hline
\end{tabular}

Table 1. Morphological features of the studied profile 
Donde $\mathrm{r}(\mathrm{m})$ corresponde al radio del pozo, $\mathrm{H}(\mathrm{m})$ a la distancia desde el nivel freático (NF) al fondo del pozo, $\mathrm{h}_{0}$ a la distancia desde el NF estabilizado al nivel del agua en el pozo al tiempo $\mathrm{t}_{0}, \mathrm{~h}_{1}$ la distancia desde el NF estabilizado al nivel del agua en el pozo al tiempo $\mathrm{t}_{1}, \Delta \mathrm{t}(\mathrm{s})$ al intervalo de tiempo entre $t_{0}$ y $_{1}$ y $S$ [-] a un factor de geometría del pozo.

En el mes de Octubre, cuando el NF se encontraba a una profundidad promedio de $120 \mathrm{~cm}$, se procedió a cavar una calicata con el fin de realizar una descripción morfológica del perfil. En esta se definieron los horizontes genéticos (Ap, Bw, BC, 2C , $2 \mathrm{C}_{2}, 2 \mathrm{C}_{3}$ ) y sus propiedades morfológicas (Cuadro 1).

Se realizaron las mediciones en terreno mediante el método del CI (cilindro infiltrómetro), y se extrajeron muestras en cilindros de hierro para realizar las mediciones con el PCC (permeameátro de carga constante) en laboratorio.

Las mediciones con el cilindro infiltrómetro (CI) se realizaron con cilindros de hierro de $50 \mathrm{~cm}$ de diámetro $x 70 \mathrm{~cm}$ de altura sobre la superficie de cada horizonte genético. La medición consistió en registrar la tasa de disminución de agua dentro del cilindro por un período máximo de 3 a 5 horas. Se construyó una curva (Figura 1) a partir de la ecuación de Kostiakov (1932):

$$
\mathrm{F}=\mathrm{at}^{\mathrm{b}} \quad[4]
$$

Donde F (cm) corresponde al flujo de entrada de agua en el suelo y está en función del tiempo t (min) y de los parámetros empíricos a y b.

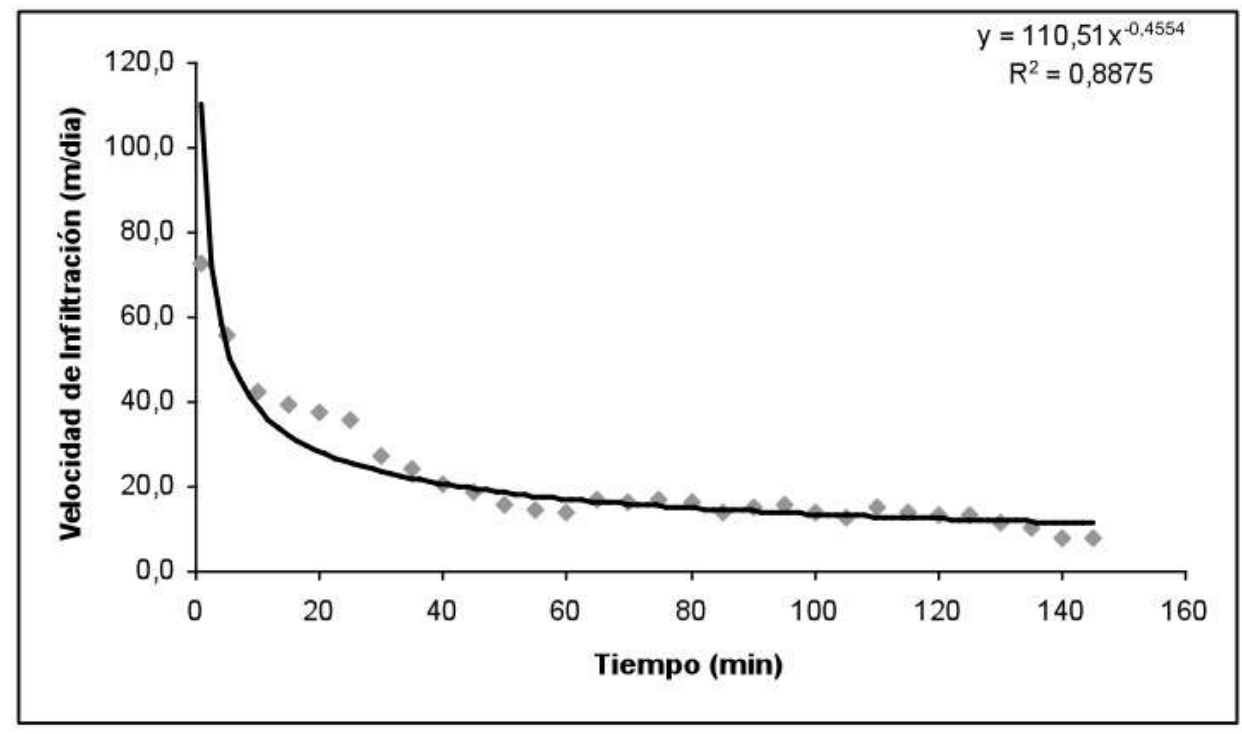

Figura 1. Curva de la Velocidad de Infiltración construída a partir de la ecuación de Kostiakov (1932).

Figure 1. Graphic of the Infiltration Rate under Kostiakov parameter. 
A partir de la ecuación [4] se asume que la velocidad de infiltración estabilizada $\left(\mathrm{VI}_{\mathrm{e}}\right)$ equivale a la $\mathrm{K}_{\mathrm{s}}$ de la sección del perfil bajo el cilindro que se encuentra saturada (Oosterbaan y Nijland, 1994; Radcliffe y Rasmussen, 2000). Se estimó ( $\mathrm{VI}_{\mathrm{e}}$ ) como el valor de velocidad donde en un intervalo de 5 minutos su variación era inferior al $1 \%$.

Para el permeámetro de carga constante (PCC), se utilizaron muestras no disturbadas, extraídas en cilindros metálicos de diámetro igual a $6 \mathrm{~cm} \times 5 \mathrm{~cm}$ de alto. Las muestras fueron tomadas en sentido vertical, para medir el vector de flujo saturado en esa dirección. De cada parcela de muestreo, correspondiente a cada repetición, se extrajeron 5 cilindros, que correspondieron a las submuestras de la repetición. Procedimiento que se realizó para cada uno de los horizontes genéticos luego de dejar despejada la superficie de estos. Las muestras fueron llevadas a laboratorio, donde se utilizó un PCC (Eijkelkamp, Holanda) para medir $\mathrm{K}_{\mathrm{s}}$. Esta se obtuvo utilizando directamente la ley de Darcy (1856):

$$
\mathrm{K}=\left(\frac{Q}{A}\right)\left(\frac{L}{\Delta H}\right)[5]
$$

Donde Q $\left(\mathrm{cm}^{3} \mathrm{~min}^{-1}\right)$ corresponde al flujo volumétrico o caudal que fluye a través del cilindro, $\mathrm{A}\left(\mathrm{cm}^{2}\right)$ y L $(\mathrm{cm})$, al área de sección $\mathrm{y}$ al largo del cilindro respectivamente, mientras que $\Delta \mathrm{H}(\mathrm{cm})$ corresponde a la carga hidráulica sobre el cilindro. Las mediciones de $\mathrm{K}_{\mathrm{s}}$ se realizaron luego de 2 horas de equilibrio dinámico en el PCC.

Debido a que los métodos del PCC y del CI entregan valores parciales de $K_{s}$, equivalente a una sección del perfil y asumida como correspondiente a cada horizonte genético, y a que el PB entrega un valor ponderado de la sección del perfil interceptada por el pozo, es que para comparar los datos entregados de las 3 metodologías analizadas, se procedió a ponderar los valores obtenidos mediante el PCC y el CI, sumando las transmisividades de la sección del perfil medida con el PB. La transmisividad total $\left(\mathrm{Dt}_{\mathrm{t}} / \mathrm{K}_{\mathrm{s}_{\mathrm{t}}}\right.$ ) de un suelo estratificado para el flujo en dirección vertical es:

$$
\frac{D_{t}}{K s_{t}}=\sum_{i=1}^{n} \frac{D_{i}}{K s_{i}}[6]
$$

Donde $\mathrm{D}_{\mathrm{i}}(\mathrm{m})$ corresponde a la potencia de la estrata y el valor $\mathrm{D} / \mathrm{Ks}$ representa la resistencia hidráulica al flujo vertical (Oosterbaan y Nijland, 1994).

\section{Análisis Estadístico}

Los tratamientos consistieron en cada método de medición de la $\mathrm{K}_{\mathrm{s}}$. La unidad experimental correspondió a la parcela de medición, a partir de la cual se estimó el valor de $\mathrm{K}_{\mathrm{s}}$ obtenido por horizonte para los métodos del CI y el PCC y al valor ponderado de la sección del perfil que entrega el método del PB. Para comparar las metodologías, en los métodos del CI y el PCC se obtuvo un valor único de Ks para el perfil de acuerdo a la fórmula [6].

Cada tratamiento contó con 3 repeticiones correspondientes a cada una de las parcelas de muestreo.

Se determinaron los estadígrafos básicos, media aritmética $(\mathrm{Kx})$, desviación estándar $(\sigma)$ y coeficiente de variación $(\mathrm{CV})$.

Se realizó una correlación entre las repeticiones de cada método mediante el uso del coeficiente de correlación de Pearson.

Finalmente, se realizó un análisis de varianza (ANDEVA) para contrastar las metodologías utilizadas. Cuando fue necesario se realizó un test de rango múltiple entre las metodologías utilizadas, a las cuales se les aplicó el test de diferencias mínimas significativas (LSD, $\alpha \leq 0,05$ ). 
Cuadro 2. Comparación de los valores de Ks ponderado del perfil según método.

Table 2. Comparisson of Ks profile values of each method.

\begin{tabular}{|c|c|c|c|c|c|}
\hline Método & $\mathbf{K x}$ & Kmáx & Kmín & $\boldsymbol{\sigma}$ & CV \\
\hline$\left[\mathrm{m} \cdot \mathbf{s}^{-1}\right]$ & \multicolumn{4}{|c|}{ - $\left[m \cdot\right.$ día $\left.^{-1}\right]$} & $\%$ \\
\hline PB & 10,77 & 15,31 & 7,54 & 4,04 & 39 \\
\hline CI & 7,10 & 7,69 & 6,04 & 0,92 & 13 \\
\hline PCC & 1,49 & 13,97 & $\mathbf{0 , 0 3}$ & 3,47 & 249 \\
\hline
\end{tabular}

\section{RESULTADOS Y DISCUSIÓN}

En el Cuadro 2, se observa que los valores de $\mathrm{K}_{\mathrm{s}}$ obtenidos mediante el método del PB son los de mayor magnitud, mientras que los valores mas bajos se obtuvieron mediante el método del PCC. Esto último se puede deber principalmente a la compactación y sellado del suelo en el cilindro durante su extracción. Mohanty et al. (1998) obtuvieron valores de $\mathrm{K}_{\mathrm{s}}$ para el PCC de un promedio de $0,24 \mathrm{~m} \mathrm{dia}^{-1}$ comparados con valores de diferentes métodos de campo cercanos a 0,7 $\mathrm{m} \mathrm{dia}^{-1}$, para un Mollisol de textura franca.

Por otra parte, el PCC integra un menor volumen de suelo, lo que se manifiesta en la mayor variabilidad de los resultados, ya que en el muestreo existe la posibilidad de integrar o excluir bioporos o grietas, aumentando o disminuyendo la Ks (Dirksen, 1999) a diferencia de los métodos del PB y CI, que incluyen un mayor volumen, aproximándose con mayor precisión al promedio del suelo.

Las diferencias obtenidas entre el PB y el CI se explicarían por la presencia de aire entrampado en el suelo durante la medición con este último método. Esta situación tiene como efecto una reducción en la sección conductora de agua, disminuyendo el valor de $K_{s}$ (Christiansen, 1944). Sin embargo esta diferencia no es considerable si se toma en cuenta que la $\mathrm{K}_{\mathrm{s}}$ es una de las propiedades más variables del suelo (Wilding, 2001).

La diferencia en los coeficientes de variación (CV) entre las distintas metodologías se puede atribuir al área de influencia de cada muestra; mientras que los cilindros para el PCC tienen un diámetro
$6 \mathrm{~cm}$ y un alto de $5 \mathrm{~cm}$, los cilindros para el CI tienen un diámetro de $50 \mathrm{~cm}$ y una altura de $70 \mathrm{~cm}$; a su vez el área de influencia del PB sería al menos del tamaño de la sección del pozo bajo el nivel freático. En el Cuadro 3 se presentan los coeficientes de correlación de Pearson entre los tres métodos estudiados. La correlación se realizó entre las repeticiones realizadas en tres puntos diferentes. El mayor ajuste entre los métodos del PB y el PCC indica que ambos estarían reflejando una tendencia similar en cuanto a la variabilidad espacial de la $\mathrm{K}_{\mathrm{s}}$. La baja correlación existente entre el PCC y el CI, se puede atribuir principalmente al mayor tamaño de la muestra de suelo en este último método, lo que lo hace menos sensible a la variabilidad espacial de la $\mathrm{K}_{\mathrm{s}}$.

$\mathrm{Si}$ bien es cierto que de los resultados del Cuadro 3 se obtiene que existe una sensibilidad similar para la variabilidad espacial entre el PB y el PCC, la diferencia en la magnitud de los datos observados entre estos métodos, junto al alto valor de CV entregado por el PCC, hace que la aplicación de éste último método lleve a dudar de su viabilidad como método de medición para este tipo de suelos.

En los cuadros 4 y 5 se entrega respectivamente el ANDEVA y el test de LSD realizado entre las $\mathrm{K}_{\mathrm{s}}$ obtenidas.

El test de diferencias mínimas significativas (LSD), arrojó que el valor de $\mathrm{K}_{\mathrm{s}}$ obtenido por el PCC es estadísticamente similar al del CI y diferente al del PB, mientras que el del CI es estadísticamente similar al del PB. 
Cuadro 3. Coeficientes de correlación de Pearson entre las metodologías analizadas.

Table 3. Pearson coefficient betwen the studied methods.

\begin{tabular}{|c|c|c|c|c|}
\hline Comparación & Valor $\mathbf{R}$ & Valor p & m & Confianza \\
\hline & & & & $\%$ \\
\hline PB v/s CI & 0,737 & 0,023 & 0,44 & 95 \\
\hline PB v/s PCC & 0,995 & 0,005 & 0,59 & 95 \\
\hline CI v/s PCC & 0,671 & 0,025 & 2,54 & 95 \\
\hline
\end{tabular}

Cuadro 4. Análisis de varianza entre métodos de medición de Ks

Table 4. ANOVA values for the Ks studied methods.

\begin{tabular}{|c|c|c|c|c|c|}
\hline & SC & Gl & CM & F & p \\
\hline $\begin{array}{c}\text { Entre } \\
\text { métodos }\end{array}$ & 102.29 & 2 & 51,14 & 5,24 & 0,049 \\
\hline
\end{tabular}

Cuadro 5. Test de Fisher LSD $(\alpha=0,05)$

Table 5. Fisher Test LSD $(\alpha=0,05)$

\begin{tabular}{|c|c|c|c|c|}
\hline Método & Media & N & \\
\hline PB & $\mathbf{1 0 , 7 7}$ & $\mathbf{3}$ & $\mathbf{A}$ & \\
\hline CI & $\mathbf{7 , 1 0}$ & $\mathbf{3}$ & $\mathbf{A}$ & $\mathbf{B}$ \\
\hline PCC & $\mathbf{1 , 4 9}$ & $\mathbf{3}$ & & $\mathbf{B}$ \\
\hline
\end{tabular}

La diferencia en el valor de $\mathrm{K}_{\mathrm{s}}$ del PCC con respecto al método del $\mathrm{PB}$, cercana a un orden de magnitud, implica que la viabilidad económica de un sistema de drenaje se pueda ver afectada, ya que un valor menor de $\mathrm{K}_{\mathrm{s}}$, implica acortar el distanciamiento entre drenes, lo que encarece la obra. Sin embargo, es necesario extender esta tipo de estudio a otros suelos para validar los resultados obtenidos en esta investigación.

\section{CONCLUSIONES}

El método del PCC presenta el menor valor de $K_{S}$ y la mayor variabilidad de los resultados, por lo que la utilización de este método para medición de $\mathrm{K}_{\mathrm{S}}$ con fines de drenaje no se recomienda para este tipo de suelos.

La menor variabilidad de Ks, determinada por el método del CI, junto con la similitud de los valores obtenidos con respecto al PB, permiten sugerir al método del CI como reemplazante del método del PB en ausencia de Nivel Freático, para suelos con características similares al estudiado.

El PCC se adaptará mejor a condiciones de medición adversa (pendiente) o para evaluar la variabilidad espacial de Ks, donde los otros dos métodos (PB y CI) no tienen tan buena adaptación. 


\section{BIBLIOGRAFÍA}

ALCAYAGA, S. 1989. Origen, distribución y caracterización de los suelos de drenaje restringido. En: Los suelos de drenaje restringido. Sociedad Chilena de la Ciencia del Suelo Boletín $N^{\circ}$ 9: $1-25$.

BOUWER, H., JACKSON, R.D. 1974. Determinig soil properties. In: J. van Schilfgaarde (Ed). Drainage for Agriculture. Agronomy 17. American Society of Agronomy, Inc. Publisher. Madison, Wisconsin, USA. p.611-672.

CHRISTIANSEN, J.E. 1944. Effect of entrapped air upon the permeability of soils. Soil Science 58: 355-365.

CIREN. 1997. Estudio Agrológico VII Región. Publicación N ${ }^{\circ} 117.659$ p.

DARCY, H. 1856. Les fontaines publiques de la ville de Dijon. Dalmont, Paris, France.

DIRSEN, C. 1999. Soil physics measurements. GeoEcology paperback. Catena Verl., Reiskirchen, Germany. $154 \mathrm{p}$.

HOOGHOUDT, S.B. 1936. Bijdragen tot de kennis van enige natuurkundige grootheden van de grond. Versl. Landb. Onderz. 42(13B) B69-B 161.

KOSTIAKOV, A.N. 1932. "On the dynamics of the coefficients of water percolation in soils and on the necessity of studying it from a dynamic point of view for purpose of amelioration". Transactions of the $6^{\text {h }}$ Communication of the Int. Society of Soil Sciences, Part A 17-21.
MAASLAND, M. 1955. Measurement of hydraulic conductivity by the auger hole method in anisotropic soil. Soil Science 81 379-388.

MOHANTY, B.P., SKAGGS, T.H., VAN GENUCHTEN, M. TH. 1998. Impact of saturated hydraulic conductivity on the prediction of tile flow. Soil Science Society of America Journal 62: 15521559.

OOSTERBAAN, R.J., NIJLAND, H.J. 1994. Determinig the saturated hydraulic conductivity.. In: Ritzema, H.P. (Ed). Drainage principles and applications. International Institute for Land Reclamation and Improvement (ILRI) Publication 16, Wageningen, The Netherlands. p. 435 - 475 RADCLIFFE, D., RASMUSSEN, T. 2000. Soil Water Movement. In: Sumner, M. (Ed.). Handbook of Soil Science. CRC Press, Boca Raton, Florida, USA. p. A87-A127.

SALGADO, L. 2000. Manual de Estándares Técnicos y Económicos para Obras de Drenaje. Comisión Nacional de Riego y Universidad de Concepción, Chillán. $415 \mathrm{p}$.

WILDING, L.P. 2001. Pedology. in: Sumner, M. (Ed.). Handbook of Soil Science. CRC Press, Boca Raton, Florida, USA. 2113p. 